\title{
Analytical solutions for the electric field and dielectrophoretic force in a dielectrophoretic focusing electrode structure
}

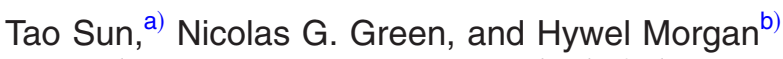 \\ Nanoscale Systems Integration Group, School of Electronics and Computer Science, \\ University of Southampton, Southampton SO17 1BJ, United Kingdom
}

(Received 15 January 2008; accepted 7 April 2008; published online 2 May 2008)

\begin{abstract}
The analysis of the movement of particles in a nonuniform field requires accurate knowledge of the electric field distribution. In this letter, the Schwarz-Christoffel mapping method is used to analytically solve the electric field distribution in a dielectrophoretic focusing electrode structure. The analytical result for the electric field distribution is validated by comparison with numerical simulations using the finite element method. The electric field solution is used to calculate the dielectrophoretic force on a particle in the system. (C) 2008 American Institute of Physics.
\end{abstract}

[DOI: $10.1063 / 1.2916827]$

Dielectrophoresis $(\mathrm{DEP})^{1-3}$ is one of the ac electrokinetic techniques where the interaction between a fieldinduced dipole moment on the particle and a nonuniform electric field produces a force on the particle. Since the dielectrophoretic forces are generated from the interaction between the electric field and the induced dipole, it is important to characterize the strength and direction of the electric field distribution in the system.

In this work, we use the Schwarz-Christoffel mapping (SCM) method ${ }^{4-7}$ to calculate the electric field generated by the dielectrophoretic focusing electrode structure shown in Fig. 1(a). By using negative DEP ( $n$ DEP), the particles are repelled from the electrode edges and focused into a tight stream in the channel center. ${ }^{8-11}$ Since the electrodes are long compared to their width, the electric field analysis can be performed through a cross section of the device, ${ }^{9}$ as shown in Fig. 1(b). Since the electrodes are much thinner than their width, they can be represented as a section of the bottom boundary at a fixed potential. The Neumann boundary condition (insulating) is used for the potential at the electrolyte/ glass interfaces. ${ }^{6,12,13}$ Applying symmetry, only a quarter of the system $(A B C D E F)$ needs to be solved in the analysis.

Figure 1(c) shows the three complex planes used for the SCM procedure. The selected cell $A B C D E F$ is rotated $90^{\circ}$ and set in the $Z$ plane with the boundary conditions for $\phi$ as shown: $\partial \phi / \partial n=0$, along the insulating walls $B C, C D$, and $E F$. Dirichlet boundary conditions define the fixed potential $\phi=V$ along the electrode $(D E)$ and the boundary for the axis of the odd symmetry, $\phi=0(A F$ and $A B)$. The interior of the polygon $A B C D E F$ in the $Z$ plane is mapped into the upper half of the $T$ plane by using the SCM method. The polygon $A B C D E F$ is opened at point $G$ and the boundaries of the polygon mapped to the real axis of the $T$ plane. The coordinates of the corresponding points $Z_{A}-Z_{F}$ in the $T$ plane are $T_{A}-T_{F}$, respectively. The point $G$ is mapped to positive and negative infinities. The SCM integral ${ }^{14}$ from the $T$ plane to the $Z$ plane is given by

\footnotetext{
${ }^{a)}$ Electronic mail: ts5@ecs.soton.ac.uk.

${ }^{b)}$ Electronic mail: hm@ecs.soton.ac.uk.
}

$$
\begin{aligned}
Z= & C_{1} \int^{T}\left(T-T_{F}\right)^{-1 / 2}\left(T-T_{C}\right)^{-1 / 2}\left(T-T_{B}\right)^{-1 / 2} \\
& \times\left(T-T_{A}\right)^{-1 / 2} d T+C_{2}
\end{aligned}
$$

where $Z=Z_{x}+j Z_{y}$ refers to the complex coordinate of any point in the interior of polygon $A B C D E F$ in the $Z$ plane. $T$ $=T_{x}+j T_{y}$ refers to the complex coordinate of any point in the upper half of the $T$ plane.

Since the SCM method allows up to three points to be arbitrarily chosen along the real axis of the $T$ plane, ${ }^{6,14}$ we fix the coordinates of $T_{A}=0, T_{B}=1$, and $T_{C}=1.5$. For $T>T_{F}$ $>T_{C}>T_{B}>T_{A}$, the solution of Eq. (1) is an elliptic integral ${ }^{15}$

$Z=C_{3} F\left(\varpi_{1}, k_{1}\right)+C_{2}=C_{3} \int_{0}^{\lambda_{1}} \frac{d \lambda_{1}}{\left(1-\lambda_{1}^{2}\right)\left(1-k_{1}^{2} \lambda_{1}^{2}\right)} d \lambda_{1}+C_{2}$,

with

$$
C_{3}=\frac{2 C_{1}}{\sqrt{\left(T_{F}-T_{B}\right)\left(T_{C}-T_{A}\right)}}
$$
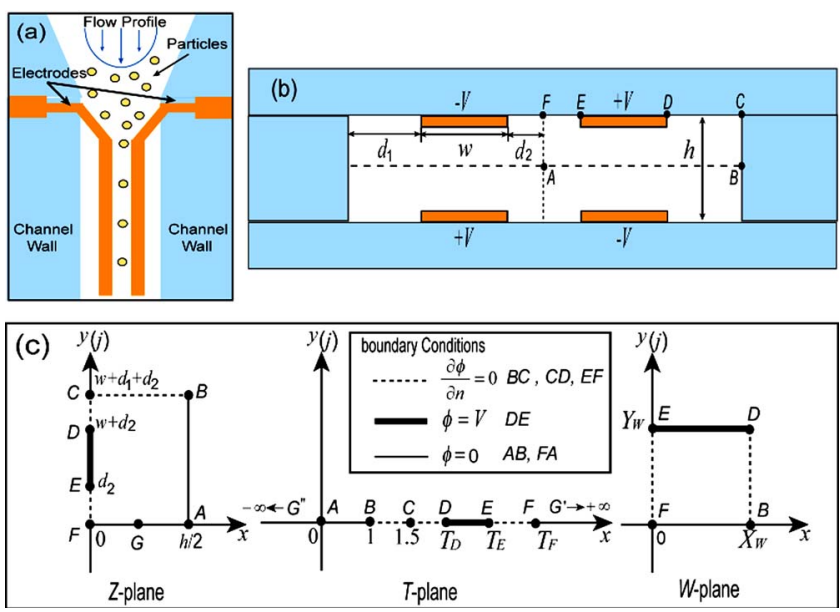

FIG. 1. (Color online) (a) Diagram showing the dielectrophoretic focusing electrodes (top view). (b) Cross section of the four focusing electrodes with the geometrical parameters. (c) The three complex planes used for SCM with corresponding boundary conditions. The Neumann boundary conditions are represented by dashed lines. The electrode is represented by a bold line. 


$$
\begin{aligned}
& \varpi_{1}=\arcsin \left[\sqrt{\frac{\left(T_{C}-T_{A}\right)\left(T-T_{F}\right)}{\left(T_{F}-T_{A}\right)\left(T-T_{C}\right)}}\right], \\
& k_{1}=\sqrt{\frac{\left(T_{C}-T_{B}\right)\left(T_{F}-T_{A}\right)}{\left(T_{F}-T_{B}\right)\left(T_{C}-T_{A}\right)}} \\
& \lambda_{1}=\sin \varpi_{1},
\end{aligned}
$$

where $F\left(\varpi_{1}, k_{1}\right)$ is the elliptical integral of the first kind and $k_{1}$ is the modulus of the elliptic function.

Equation (2) links the $T$ plane to the $Z$ plane. The values of the coefficients $C_{2}$ and $C_{3}$ can be solved by a mapping relationship between the coordinates of the corresponding points in the two planes $\left(Z_{F} \rightarrow T_{F}, Z_{C} \rightarrow T_{C}, Z_{B} \rightarrow T_{B}, Z_{A}\right.$ $\left.\rightarrow T_{A}\right)$

$$
\begin{aligned}
& C_{2}=0, \\
& C_{3}=\frac{h}{2 K\left(k_{1}\right)}=\frac{d_{1}+w+d_{2}}{K\left(k_{1}^{\prime}\right)},
\end{aligned}
$$

where $K\left(k_{1}\right)$ is the complete elliptic integral of the first kind and $k_{1}^{\prime}=\sqrt{1-k_{1}^{2}}$. Equation (4b) also provides the relationship between the complete elliptical integral and the geometrical parameters of the system

$$
\frac{K\left(k_{1}\right)}{K\left(k_{1}^{\prime}\right)}=\frac{h}{2\left(d_{1}+w+d_{2}\right)} .
$$

The value of the modulus $k_{1}$ can be calculated by inputting arbitrary geometrical parameters from Hilberg's approximation. ${ }^{16}$ Then, from Eq. (3c), the value of $T_{F}$ is given by

$$
T_{F}=\frac{T_{B}\left(T_{C}-T_{A}\right) k_{1}^{2}-T_{A}\left(T_{C}-T_{B}\right)}{\left(T_{C}-T_{A}\right) k_{1}^{2}-\left(T_{C}-T_{B}\right)} .
$$

The inverse function of Eq. (2) enables us to express $T$ in terms of $Z$,

$$
T=\frac{T_{F} T_{C} \operatorname{cn}^{2}\left(\frac{Z}{C_{3}}, k_{1}\right)}{T_{C}-T_{F} \operatorname{sn}^{2}\left(\frac{Z}{C_{3}}, k_{1}\right)},
$$

where $\operatorname{sn}(\ldots, \ldots)$ and $\mathrm{cn}(\ldots, \ldots)$ are the Jacobian elliptic functions.

The coordinate of points $D$ and $E$ in the $T$ plane can be obtained from Eq. (7),

$$
\begin{gathered}
T_{D}=\frac{T_{F} T_{C} \operatorname{cn}^{2}\left(j \frac{w+d_{2}}{C_{3}}, k_{1}\right)}{T_{C}-T_{F} \operatorname{sn}^{2}\left(j \frac{w+d_{2}}{C_{3}}, k_{1}\right)}, \\
T_{E}=\frac{T_{F} T_{C} \operatorname{cn}^{2}\left(j \frac{d_{2}}{C_{3}}, k_{1}\right)}{T_{C}-T_{F} \operatorname{sn}^{2}\left(j \frac{d_{2}}{C_{3}}, k_{1}\right)} .
\end{gathered}
$$

The second SCM is used to transform the upper half of the $T$ plane into a rectangle in the model plane ( $W$ plane). The electric field is uniformly distributed in the interior of the rectangle due to the restriction from the transformed bound- aries in the $W$ plane. The corresponding points are $W_{E}$ $=j Y_{W}, W_{D}=X_{W}+j Y_{W}, W_{B}=X_{W}$, and $W_{F}=0$, where $X_{W}$ and $Y_{W}$ are the size of the rectangle along the real and imaginary axes, respectively. Similarly, the transformation from the $T$ plane to the $W$ plane is given by

$$
\begin{aligned}
W= & D_{1} \int^{T}\left(T-T_{F}\right)^{-1 / 2}\left(T-T_{E}\right)^{-1 / 2}\left(T-T_{D}\right)^{-1 / 2} \\
& \times\left(T-T_{B}\right)^{-1 / 2} d T+D_{2} .
\end{aligned}
$$

The integral solution of Eq. (9) is

$W=D_{3} F\left(\varpi_{2}, k_{2}\right)+D_{2}=D_{3} \int_{0}^{\lambda_{2}} \frac{d \lambda_{2}}{\left(1-\lambda_{2}^{2}\right)\left(1-k_{2}^{2} \lambda_{2}^{2}\right)} d \lambda_{2}+D_{2}$,

with

$$
\begin{aligned}
& D_{3}=\frac{2 D_{1}}{\sqrt{\left(T_{F}-T_{D}\right)\left(T_{E}-T_{B}\right)}}, \\
& \varpi_{2}=\arcsin \left(\sqrt{\frac{\left(T_{E}-T_{B}\right)\left(T-T_{F}\right)}{\left(T_{F}-T_{B}\right)\left(T-T_{E}\right)}}\right), \\
& k_{2}=\sqrt{\frac{\left(T_{E}-T_{D}\right)\left(T_{F}-T_{B}\right)}{\left(T_{F}-T_{D}\right)\left(T_{E}-T_{B}\right)}}, \quad \lambda_{2}=\sin \varpi_{2} .
\end{aligned}
$$

Equation (10) links the $T$ plane with the $W$ plane. The values of the coefficients $D_{2}$ and $D_{3}$ can be obtained by a similar mapping procedure to the first SCM performance,

$$
D_{2}=0, \quad D_{3}=\frac{X_{W}}{K\left(k_{2}\right)}=\frac{Y_{W}}{K\left(k_{2}^{\prime}\right)} .
$$

Since Laplace's equation remains invariant under conformal mapping, the potential gradients in the physical plane $\nabla \phi_{Z}$ and model plane $\nabla \phi_{W}$ are related by ${ }^{14}$

$$
\nabla \phi_{Z}=\nabla \phi_{W} \overline{f^{\prime}(Z)}=\nabla \phi_{W} \frac{\overline{d W}}{d Z} .
$$

$\overline{f^{\prime}(Z)}$ is the conjugate of the derivative of $f(Z)$, which is the linking transformation equation between the $Z$ and $W$ planes. By using this relationship and combining Eqs. (1) and (10), the nonuniform electric field distribution in the $Z$ plane, $\mathbf{E}_{Z}$, can be derived as

$\mathbf{E}_{Z}=j \frac{2 \frac{V}{h} \frac{K\left(k_{1}\right)}{K\left(k_{2}^{\prime}\right)} \frac{\left(T_{F}-T_{D}\right)^{1 / 2}\left(T_{E}-T_{B}\right)^{1 / 2}\left(T-T_{A}\right)^{1 / 2}\left(T-T_{C}\right)^{1 / 2}}{\left(T_{F}-T_{B}\right)^{1 / 2}\left(T_{C}-T_{A}\right)^{1 / 2}\left(T-T_{D}\right)^{1 / 2}\left(T-T_{E}\right)^{1 / 2}}}{,}$

where $V$ is the potential difference between the electrode DE and the zero potential $B F$.

Equation (13) is the analytical solution for the electric field in the chosen area $A B C D E F$. The features of the electric field distribution that are governed by the geometry of the device are clearly identified. The electric field magnitude approaches zero at points $A$ and $C$ (when $T=T_{A}$ and $T=T_{C}$ ) and infinity at points $D$ and $E$ (when $T=T_{D}$ and $T=T_{E}$ ), the edge of the electrode.

Numerical calculations [of Eq. (13)] were used to plot the field distribution by using MATLAB ${ }^{\mathrm{TM}}$ (Mathworks Inc., USA). The geometrical parameters are identical with the device reported in Ref. 9: $h=10 \mu \mathrm{m}, w=30 \mu \mathrm{m}, d_{1}=20 \mu \mathrm{m}$, AIP license or copyright; see http://apl.aip.org/apl/copyright.jsp 


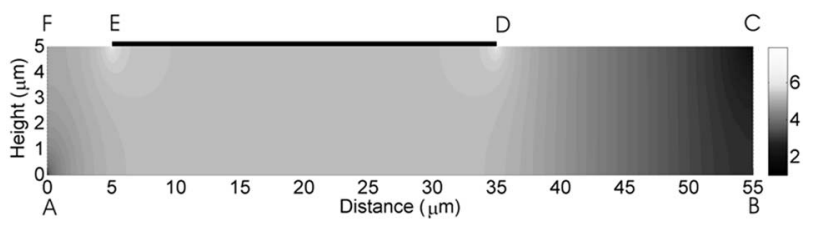

(a)

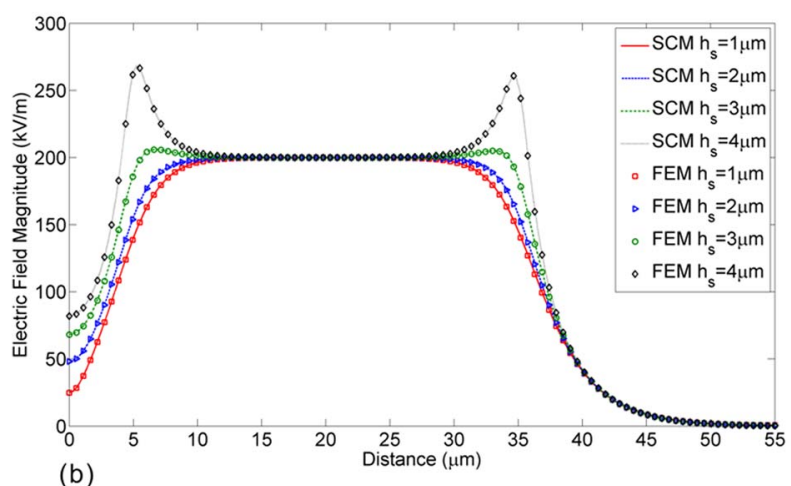

FIG. 2. (Color online) (a) Magnitude of the electric field (V/m, $\log _{10}$ scale) within the chosen area $A B C D E F$. The electrode position is drawn in the figure. (b) Electric field magnitude plotted along horizontal lines at chosen heights above line $\mathrm{AB}$. Lines are data from SCM, symbols are data from FEMLAB (FEM).

and $d_{2}=5 \mu \mathrm{m}$ and the voltage on the electrode is set to $1 \mathrm{~V}$. Figure 2(a) shows a two-dimensional plot of the magnitude of the electric field within the analyzed area $A B C D E F$. The field magnitude sharply increases toward the edges of the electrodes, the fringing field. The low electric field region can be clearly observed. In order to validate the results, the analytical solutions were compared with the numerical simulations performed by using the finite element method (FEM) in FEMLAB ${ }^{\mathrm{TM}}$ (Comsol, Ltd.). The electric field magnitude was sampled along the horizontal lines at several heights above the line $A B$, as shown in Fig. 1(b). Figure 2(b) shows excellent agreement between the SCM solution and FEM. Note that when approaching the electrode $\left(h_{s}=4 \mu \mathrm{m}\right)$, the fringing field becomes pronounced.

From the dipole approximation, the time-averaged dielectrophoretic force on a spherical particle is ${ }^{2}$

$$
\left\langle\mathbf{F}_{\mathrm{DEP}}\right\rangle=\pi \varepsilon_{m} R^{3} \operatorname{Re}\left[\frac{\widetilde{\varepsilon}_{p}-\widetilde{\varepsilon}_{m}}{\widetilde{\varepsilon}_{p}+2 \widetilde{\varepsilon}_{m}}\right] \nabla|\mathbf{E}|^{2},
$$

where $R$ is the radius of the particle. $\varepsilon_{m}$ is the permittivity of the medium. $\widetilde{\varepsilon}_{p}$ and $\widetilde{\varepsilon}_{m}$ are the complex permittivity of the particle and medium, respectively.

In order to calculate the DEP force, the size of the particle and the dielectric properties of the particle and the medium must be known, together with the electric field energy density $\nabla|\mathbf{E}|^{2}$, which is determined from the analytical electric field solution. ${ }^{6}$

Figure 3(a) is the vector plot of the factor $\nabla|\mathbf{E}|^{2}$ in the case of $n \mathrm{DEP}$, for which the particle is less polarizable than the medium. Particles experiencing $n \mathrm{DEP}$ will be pushed toward points $A$ and $C$, where the electric field magnitude is the lowest. This plot is identical to that obtained from nu-
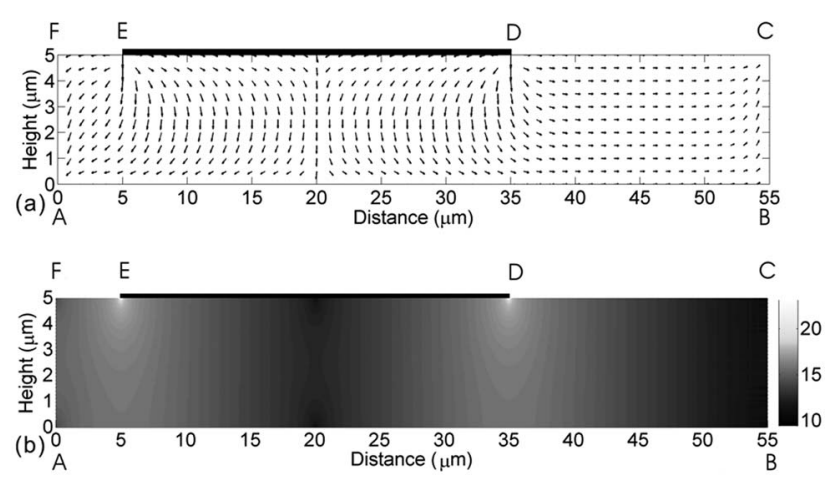

FIG. 3. Plot of $n$ DEP force component $\left(\nabla|\mathbf{E}|^{2}\right)$ in the rectangle $A B C D E F$. (a) Direction vectors and (b) magnitude $\left(\mathrm{V}^{2} / \mathrm{m}^{3}, \log _{10}\right.$ scale).

merical simulation. ${ }^{9}$ In the experiments, particles flow through the channel in the gap between two adjacent electrodes. Therefore, particles are pushed toward point $A$, focusing into a stream. Figure $3(\mathrm{~b})$ is a plot of the magnitude of $\nabla|\mathbf{E}|^{2}$ showing the maximum at the electrode edge. In this geometry, the $n$ DEP force is weak in the midpoint of the electrode and also near the channel wall. Also, the existence of these two regions ensures that particles are focused into the stream through point $A$ rather than moving to point $C$.

In this letter, we derived the analytical solutions of the electric field and dielectrophoretic force in the general DEP focusing electrodes. The results are consistent with the numerical simulations and can be applied to any geometry of this type of design. The moving trajectory of the particles can then be accurately characterized by combining the DEP force and other forces (e.g., hydrodynamic drag force and gravitational force) in the system.

The work is partly supported by the funding from the Life Science Initiative of the University of Southampton.

${ }^{1}$ H. A. Pohl, Dielectrophoresis (Cambridge University Press, New York, 1978).

${ }^{2}$ H. Morgan and N. G. Green, AC Electrokinetics: Colloids and Nanoparticles (Research Studies, Baldock, Hertfordshire, England, 2003).

${ }^{3}$ J. Voldman, Annu. Rev. Biomed. Eng. 8, 425 (2006).

${ }^{4} \mathrm{~S}$. Gevorgian, H. Berg, H. Jacobsson, and T. Lewin, IEEE Microw. Mag. 4, 60 (2003).

${ }^{5}$ T. Sun, N. G. Green, S. Gawad, and H. Morgan, IET Nanobiotechnology 1, 69 (2007).

${ }^{6}$ T. Sun, H. Morgan, and N. G. Green, Phys. Rev. E 76, 046610 (2007).

${ }^{7}$ R. Miller, Y. Y. Lau, and J. H. Booske, Appl. Phys. Lett. 91, 074105 (2007).

${ }^{8}$ S. Fielder, S. G. Shirley, T. Schnelle, and G. Fuhr, Anal. Chem. 70, 1909 (1998).

${ }^{9}$ H. Morgan, D. Holmes, and N. G. Green, IEE Proc.: Nanobiotechnol. 150, 76 (2003).

${ }^{10}$ T. Sun, S. Gawad, C. Bernabini, N. G. Green, and H. Morgan, Meas. Sci. Technol. 18, 2859 (2007).

${ }^{11}$ T. Sun, D. Holmes, S. Gawad, N. G. Green, and H. Morgan, Lab Chip 7, 1034 (2007).

${ }^{12}$ N. G. Green, A. Ramos, A. González, A. Castellanos, and H. Morgan, J. Electrost. 53, 71 (2001).

${ }^{13}$ N. G. Green, A. Ramos, and H. Morgan, J. Electrost. 56, 235 (2002).

${ }^{14}$ R. Schinzinger and P. A. A. Laurra, Conformal Mapping: Methods and Applications (Dover, Mineola, New York, 2003).

${ }^{15}$ I. S. Gradshteyn and I. M. Ryzhik, Table of Integrals, Series and Products, 5th ed. (Academic, San Diego, New York, 1994).

${ }^{16}$ W. Hilberg, IEEE Trans. Microwave Theory Tech. 17, 259 (1969). 